\title{
Experimentally determined distribution of granular-flow characteristics in collisional bed load transport
}

\author{
Václav Matoušek ${ }^{1, *}$, Štěpán Zrostlík ${ }^{1}$, Luigi Fraccarollo $^{2}$, Anna Prati $^{2}$, and Michele Larcher ${ }^{3}$ \\ ${ }^{1}$ Czech Technical University in Prague, Faculty of Civil Engineering, 16629 Prague 6, Czech Republic \\ ${ }^{2}$ University of Trento, Department of Civil, Environmental and Mechanical Engineering, Trento, Italy \\ ${ }^{3}$ Free University of Bozen-Bolzano, Department of Science and Technology, Bolzano, Italy
}

\begin{abstract}
A series of laboratory experiments on turbulent open-channel two-phase flow in a form of intense bed load transport is reported. Measurements in a laboratory tilting flume included camera based imaging techniques to identify the structure of the flow at the local level. Obtained experimental distributions of two-phase flow related parameters - granular velocity, concentration, and temperature - across a collisional transport layer are discussed. The results are analysed together with additional measured quantities (discharges of mixture and grains, flow depth, bed slope etc). Our major goal is to evaluate the distribution of granular stresses across the transport layer with a special attention paid to the interface between the transport layer and the bed. Furthermore, comparisons are discussed between the experimental results and predictions produced by suitable kinetic-theory based models.
\end{abstract}

\section{Introduction}

Intense bed load transport is associated with flood discharges in steep-slope mountain torrents and rivers. It can be also generated by transient flows, e.g. dam-break flows, in a channel with a mobile bed. The liquid- and solid phases interact in a complex way within a bed load layer developed above a mobile bed. Transported sediment grains are supported by mutual contacts, primarily collisions, and by residual turbulent uplift. It is poorly understood how to describe mechanisms that interrelate parameters like the sediment discharge and the flow depth. The parameters are dependent on distributions of velocity and concentration of solid grains in the flow and hence also on distributions of normaland shear stresses by solids.

Principles of granular rheology as expressed in the granular kinetic theory [1-2] should serve to model such flows. Kinetic theory based models are available for various granular flow conditions and geometries [3-5]. However, for open channel bed load flows with typically large gradients of local concentrations and velocities the models have not been sufficiently verified by experiments yet. The goal of our work is to produce reliable experimental data suitable to evaluate predictive abilities of the kinetic theory based models. It means that granular flow must be observed on the local level enabling to identify vertical distributions of grain concentration and velocity in a shear layer through which bed load is transported above the bed.

\section{Experimental work}

We present experiments carried out in the Water Engineering Laboratory of Czech Technical University in Prague in June 2016.

\subsection{Flow conditions}

We study turbulent two-phase flow under the following conditions:

- gravity-driven open-channel flow, steady-state uniform flow;

- flow over mobile bed at upper-stage plane bed regime (high bed shear);

- transported sediment grains supported by mutual contacts, no turbulent suspension;

- broad range of bed slopes, flows depths, sediment flow rates, and total flow rates.

For the experiment presented in this paper, we used one fraction of plastic grains (mass-median diameter 3.18 $\mathrm{mm}$, density $1358 \mathrm{~kg} / \mathrm{m}^{3}$, terminal settling velocity 0.131 $\mathrm{m} / \mathrm{s}$, round shape of grains) as model sediment in order to ensure intense collisional transport and create suitable conditions for particle tracking by cameras.

* Corresponding author: v.matousek@,fsv.cvut.cz 


\subsection{Experimental set up and measurement techniques}

\subsubsection{Tilting flume}

We used our recirculating flume with a rectanular cross section. It is $8 \mathrm{~m}$ long, $0.20 \mathrm{~m}$ wide, $0.27 \mathrm{~m}$ deep. It is equipped for continuous measurement of flow rate of carrying liquid and transported solids along the closed circuit. In the flume, a position of flow surface is sensed in 5 measuring cross sections along the length of the flume. The flume and its permanent measuring equipment (magnetic flowmeter, differential pressure transducers, water surface acoustic sensors, flume inclination meter etc.) are described in detail elsewhere [6].

\subsubsection{Camera based measuring techniques}

We used camera based measuring techniques which were developed and used (in cooperation with other researchers) in the laboratory of University in Trento.

Laser stripe technique [7] using a camera capturing a deformation of a laser strip (Figure 1) is used to determine a vertical distribution of concentration of grains across a collisional transport layer. It covers local solids concentration within a broad range of values ( 0 to 0.6 by volume) in the near-wall region of channel flow and compensates for the effect of a presence of the transparent wall on the local distribution of the grains.

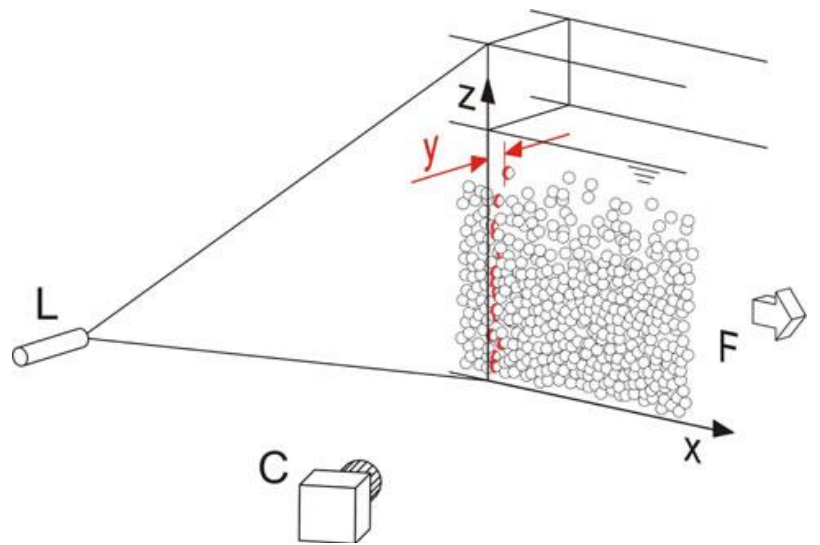

Fig. 1. Schematic sketch of set for concentration distribution measurement using laser stripe method [7].

To measure local velocities of colliding grains in time and space we adopted a Voronoi imaging technique based on Particle Tracking Velocimetry [8] using two synchronized high-speed cameras (resolution $1024 \times$ 1024 pixels, scale $\approx 3$ pixels $/ \mathrm{mm}$, frame rate $500 \mathrm{~Hz}$ over duration $3.07 \mathrm{~s}$ ) (Figure 2). The method captures three components of local velocity of colliding grains and enables to conduct statistics of velocity fluctuations.

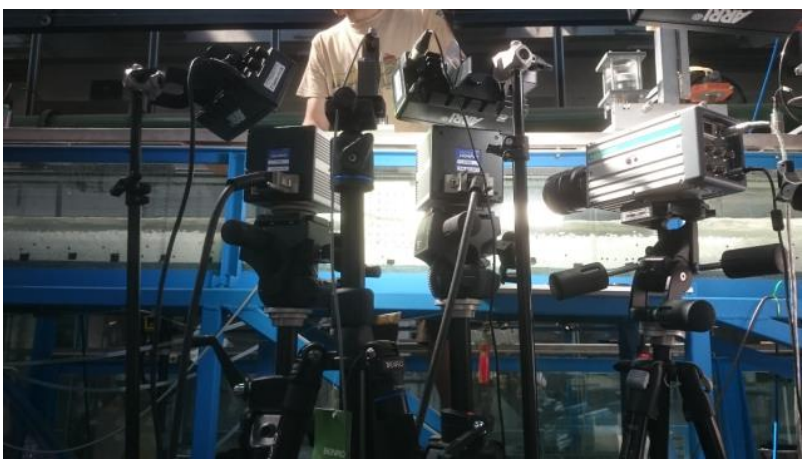

Fig. 2. Stereo camera set for velocity distribution measurement using imaging technique.

\subsection{Experimental results}

\subsubsection{Global quantities}

We carried out 4 test runs at approximately constant flow rate of mixture and 4 different bed slopes (the flume was tilted to 4 different angles starting at low angle for Run 1 and reaching the steepest slope for Run 4). As a result we obtained different solids flow rates (expressed here as the transport concentration defined as the ratio of the volumetric flow rate of grains and of the total volumetric flow rate) and corresponding bed shear stresses (expressed by the dimensionless Shields parameter). Table 1 summarizes values of the global quantities of flow and shows that both the transport concentration and the Shields parameters increased with the steeper installed slope.

Table 1. Major global quantities of flow by test runs.

\begin{tabular}{|c|c|c|c|c|}
\hline Quantity & Run 1 & Run 2 & Run 3 & Run 4 \\
\hline $\begin{array}{c}\text { Flow velocity } \\
(\mathrm{m} / \mathrm{s})\end{array}$ & 0.59 & 0.71 & 0.77 & 0.73 \\
\hline $\begin{array}{c}\text { Flow depth } \\
(\mathrm{mm})\end{array}$ & 54 & 49 & 45 & 46 \\
\hline $\begin{array}{c}\text { Bed slope } \\
(-)\end{array}$ & 0.011 & 0.017 & 0.025 & 0.036 \\
\hline $\begin{array}{c}\text { Transport } \\
\text { concentration (-) }\end{array}$ & 0.013 & 0.033 & 0.062 & 0.118 \\
\hline $\begin{array}{c}\text { Shields } \\
\text { parameter (-) }\end{array}$ & 0.48 & 0.68 & 0.92 & 1.43 \\
\hline $\begin{array}{c}\text { Froude number } \\
(-)\end{array}$ & 0.80 & 1.03 & 1.16 & 1.09 \\
\hline
\end{tabular}

A detailed analysis of flows carrying the plastic grains including the fraction tested here was published recently [9]. The analysis evaluates also an interesting change in a trend in a relation between the flow depth and the bed slope (the depth no longer decreases with the increasing slope if the Shields parameter exceeds a threshold value of about 1). Now we focus on measured local flow quantities and their relationship to the global quantities. 


\subsubsection{Distribution of concentration of grains}

The measured distribution of the local volumetric concentration of grains is plotted in Figure 3. The local concentration, $c$, is normalized by the maximum concentration, $c_{\max }$, which is the bed concentration and is considered 0.65 here). The concentration is plotted against the relative vertical position within the collisional transport layer, $y$, normalized by the thickness of the collisional layer, $h_{\text {coll }}$.

The distribution seems to be approximately linear with a very similar slope for the test runs $1-3$, i.e. for runs at the Shields parameters below a value of one, although there might be a slight difference in local concentrations just above the bottom of the collisional layer. The distribution of the test run 4 exhibits a considerably lower slope and reaches higher local values of the concentration near the bottom of the collisional layer. This observation is consistent with our analysis of the earlier experiments [10].

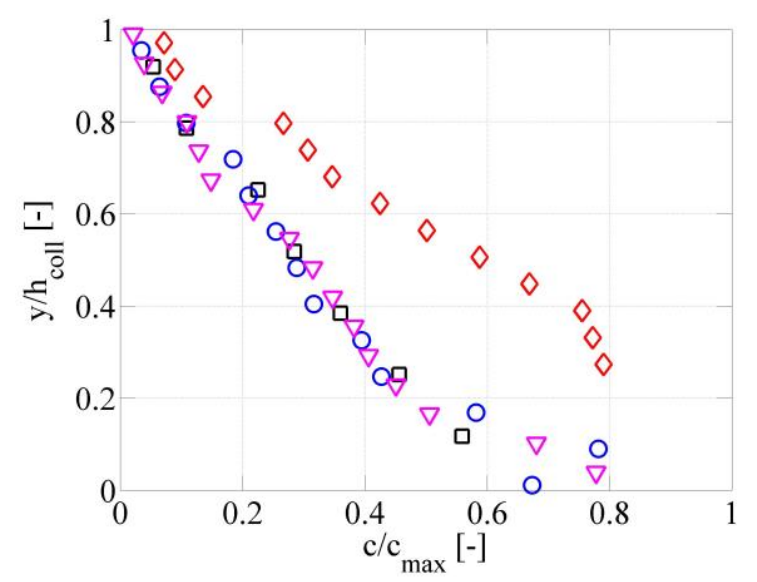

Fig. 3. Measured vertical distribution of volumetric concentration of grains across collisional layer (expressed as relative value to maximum concentration $\left.\mathrm{c}_{\max }=0.65\right)$. Legend: black square - Run 1, blue circle - Run 2, magenta triangle Run 3, red diamond - Run 4.

\subsubsection{Distribution of velocity of grains}

The measured distribution of the local velocity of grains is plotted in Figure 3. The local solids velocity, $u_{s}$, is normalized in the way used in the kinetic theory (e.g. [1]).

The distribution seems to be approximately linear for all test runs. The slope tends to increase with the increasing Shields parameter provided that the Shields does not exceed a value of one. For the Shields above one, the slope does not further increase and remains approximately constant, i.e. independent of the Shields parameter.

This observation is consistent with our experimental results obtained for a distribution of velocities in the collisional transport layer by acoustic methods [10].

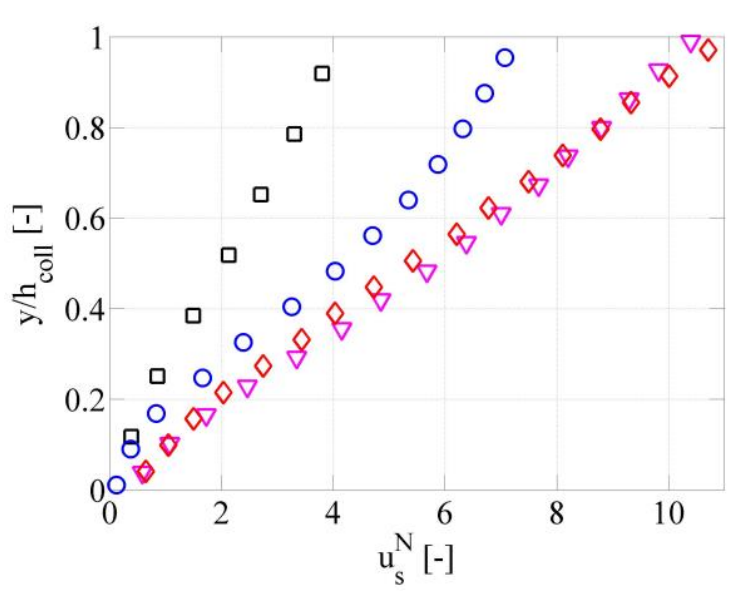

Fig. 4. Measured vertical distribution of velocity of grains across collisional layer (expressed in dimensionless form). Legend: black square - Run 1, blue circle - Run 2, magenta triangle - Run 3, red diamond - Run 4.

\subsubsection{Distribution of granular temperature}

The granular temperature is a quantity expressing a kinetic energy of intergranular collisions [1]. It is obtained from the stereo camera measurements of local granular velocities by applying a statistical method which determines the fluctuating component of the local velocity of a colliding grain in all three directions.

In Figure 5, the dimensionless granular temperature (it is made dimensionless in the way the kinetic theory applies, e.g. [1]) is plotted as a function of the relative vertical position within the collisional layer.

The measurements reveal an interesting trend in a development of the distribution of the granular temperature across the collisional transport layer. While the temperature gradually increases from the approximately zero at the bottom of the colliding layer to a certain maximum at the top of the layer in flows at low Shields parameter, in flows of high Shields parameter the maximum value is reached within the layer (Figure $5)$.

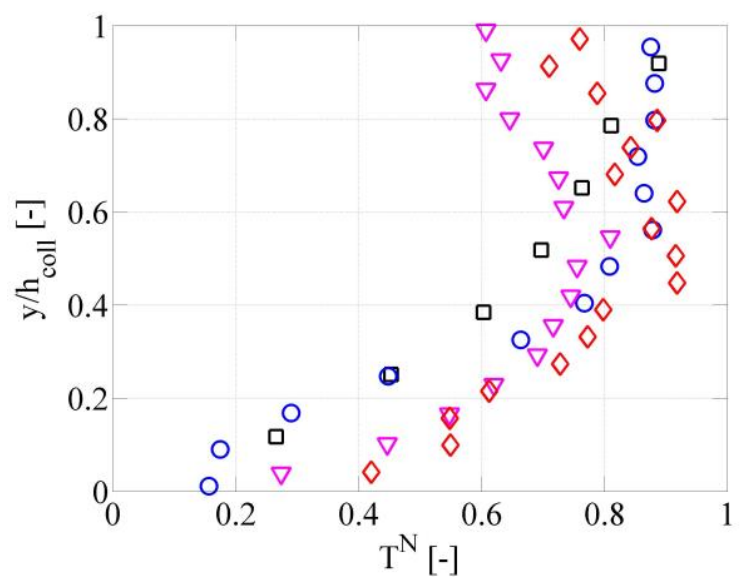

Fig. 5. Measured vertical distribution of granular temperature across collisional layer (expressed in dimensionless form). Legend: black square - Run 1, blue circle - Run 2, magenta triangle - Run 3, red diamond - Run 4. 


\section{Analysis and discussion}

\subsection{Distribution of granular stresses}

Kinetic theory for granular flows offers constitutive relations which relate depth distributions of granular stresses with depth distributions of concentration and velocity in solid-fluid flows dominated by granular collisions. Distributions produced by the constitutive relations have not yet been verified experimentally for bed-load transport.

We use our experimental data (measured profiles estimated for convenience as linear profiles) in order to produce granular stress distributions using kinetic-theory based relations. A procedure for calculating the stresses from the test based vertical profiles of granular velocity and concentration is described in [11].

To summarize the procedure, momentum balance equations are employed to get the distributions of the total shear stress and of the solids normal stress across the collisional layer. The force balance between the driving force and the resisting force assumes that the total shear stress $s+S$ (composed of the granular component, $s$, and the liquid component, $S$ ) at each vertical position, $y$, balances the longitudinal component of the weight of overlaying burden of liquid and solids. The granular normal stress is supposed to balance the normal component of the submerged weight of grains above the vertical position y. However, the momentum balance does not directly produce the granular shear stress corresponding with the granular normal shear stress. The kinetic theory must be employed to find this relationship. In the kinetic theory, the local granular stresses (normal and shear components) depend on grain density, velocity gradient (strain rate), and granular temperature. Relationships based on the classical kinetic theory contain no empirical constants. Measured distributions of granular velocity and concentration are used to calculate the distribution of the shear stresses by grains using kinetic theory based equations. An assumption of a negligible contribution of the fluid component of the total shear stress at the bottom of the collisional layer enables to check the equality of the local total shear stress by the momentum balance with the granular shear stress by a kinetic theory model at this boundary. Hence, predicted value of solids shear stress at the bottom of the collisional transport layer is an appropriate indicator of quality of a prediction of the solids shear stress by a kinetic theory based model.

Different kinetic theory based models used in the evaluation $[1,12,13]$ give a slightly different distributions of the solids shear stress, but there is generally a good agreement between the predictions of the granular shear stress and the measurement derived total shear stress at the bottom of the collisional layer provided that the Shields parameter of the flow is lower than the threshold value of about one (Figure 6). No reasonable agreement is found if the Shields parameter exceeds the threshold value (Figure 7). This seems to confirm that classical kinetic theory works well for collision-dominated flows of low to moderate local concentrations but fails at higher concentrations where granular collisions are no longer uncorrelated. In flows exhibiting the dimensionless bed shear stress higher than one, the local concentration in the vicinity of the bottom of the transport layer approaches $c_{\max }$ and the develops the dense sliding layer meaning that flow conditions are unsuitable for applying principles of the classical kinetic theory.
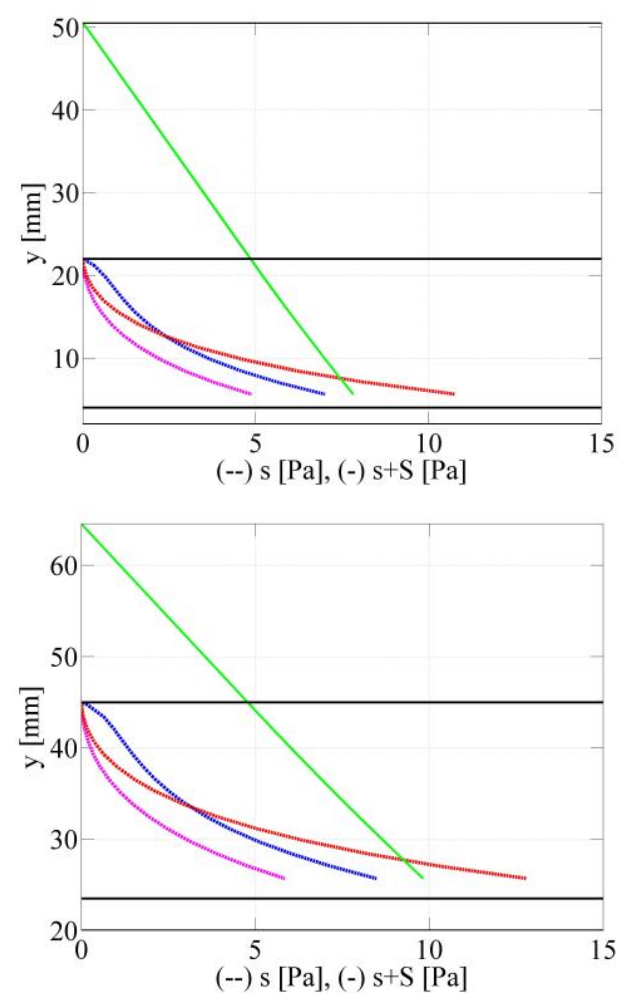

Fig. 6. Distribution of total shear stress $(\mathrm{s}+\mathrm{S})$ and solids shear stress (s), solids stress in calculated by kinetic-theory based methods for flows of low Shields parameter (up = Run 2, down $=$ Run 3). Legend: green - distribution of total shear stress; blue - distribution of solids shear stress by [1]; magenta - distribution of solids shear stress by [12]; red - distribution of solids shear stress using the method by [13]; black = positions of visually observed interfaces (bottom and top of transport layer, water surface).

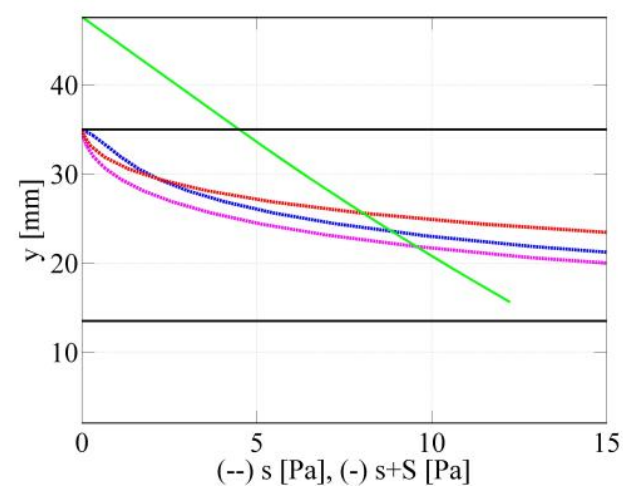

Fig. 7. Distribution of total shear stress $(\mathrm{s}+\mathrm{S})$ and solids shear stress (s), solids stress in calculated by kinetic-theory based methods for flow of high Shields parameter (Run 4). Legend: as in Fig. 6. 


\subsection{Comparison with other experiments}

Similar experiments with intense bed load transport of plastic grains was carried out in a laboratory flume at University of Trento in 2009 [13]. The same optical techniques were adopted to sense and analyse the granular distributions in an observed collisional layer.

Plots in Figures 8 and 9 compare the Prague experimental results with the Trento results for similar flow conditions (values of Shields parameter). The plots show results in the entire sensed range of vertical positions including positions below and above the collisional layer where the results are actually not valid.
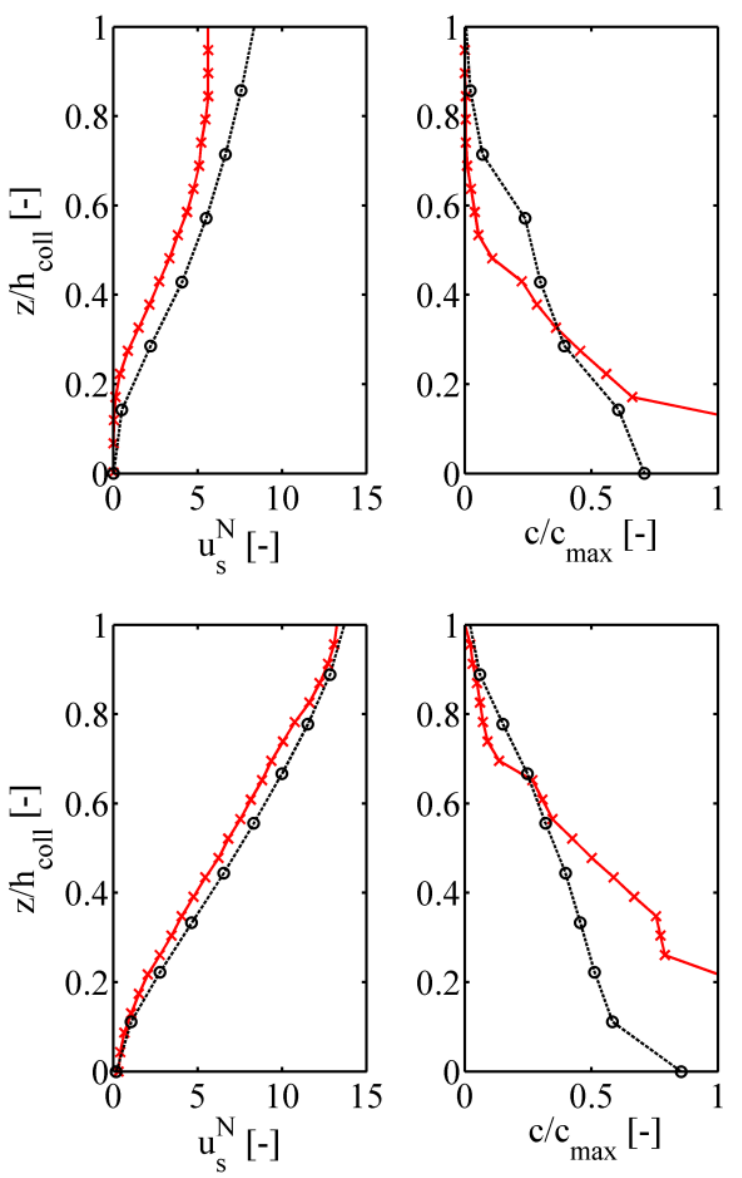

Fig. 8. Distribution of granular velocity and concentration across flow depth (up = Run 1 , down $=$ Run 4). Legend: red cross - 2016 Prague tests; black circle - 2009 Trento tests.

In the collisional layer, the agreement between the results of the two tests is satisfactory. Higher quality of camera images was reached in the Prague tests which enabled to more accurate and reliable processing of the velocity fluctuations and hence the granular temperatures than in the Trento tests (the temperature results of the Trento tests are published here for the first time).
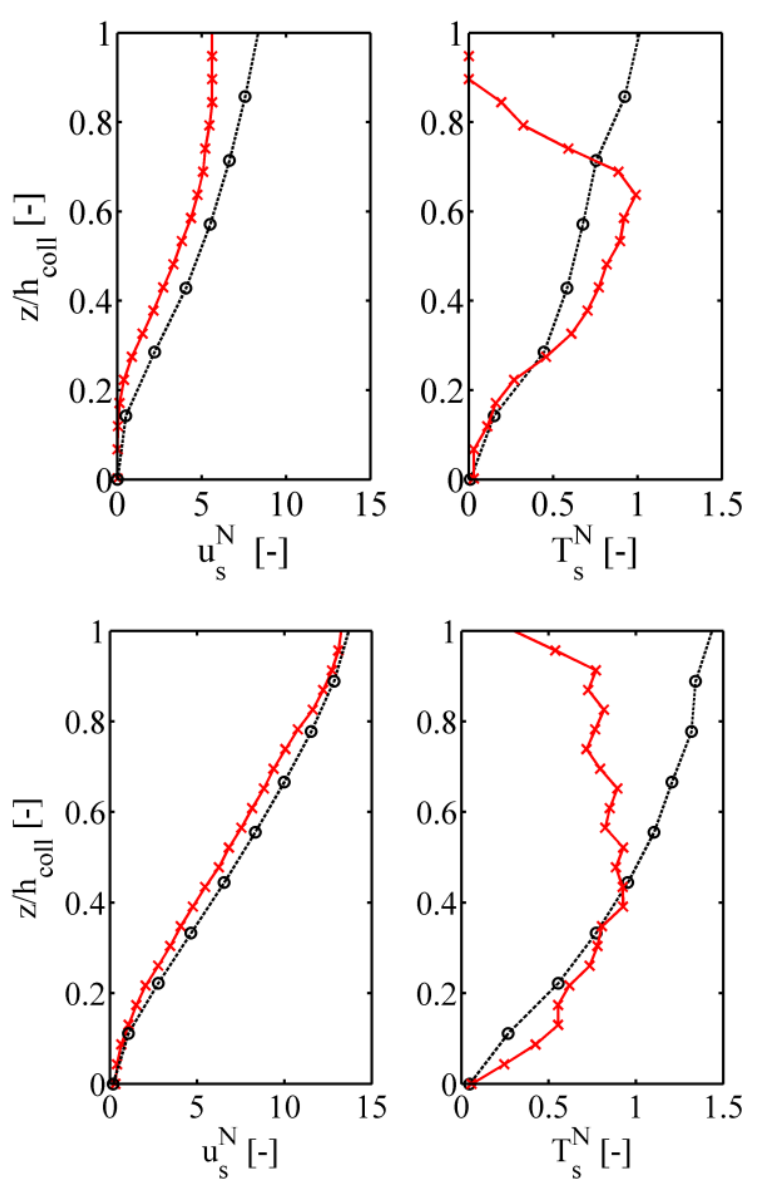

Fig. 9. Distribution of granular temperature across flow depth (up $=$ Run 1, down $=$ Run 4). Legend: as in Figure 8.

\section{Conclusions}

Kinetic theory based predictions of depth distribution of granular shear stress are evaluated using results of our contact-load experiments and assuming linear profiles of grain concentration and velocity across the collisional layer.

Solids stress at the bottom of the collisional layer resulting from the predicted distribution agreed well with the experimentally determined total shear stress at the same interface (provided that the bed Shields parameter was lower than say 1).

The classical kinetic theory works well for collisiondominated flows of low to moderate local concentrations where granular collisions are uncorrelated.

The granular temperature tends to reach a maximum value near the centre of the collisional layer thickness provided that the thickness (and hence the Shields parameter) is high enough to develop sufficiently high local concentration at the bottom of the collisional layer. Otherwise, the maximum of the temperature occurs near the top of the collisional layer. 


\section{Acknowledgement}

The research has been supported by the Czech Science Foundation through the grant project No. 16-21421S and by Faculty of Civil Engineering of the Czech Technical University in Prague through the student grant project No. SGS17/065/OHK1/1T/11. An assistance of V. Bareš, Z. Chára, J. Hlom, J. Krupička, and T. Picek with the Prague experiments is highly acknowledged.

\section{References}

1. J.T. Jenkins, D.M. Hanes, J. Fluid Mech. 370, 29-52 (1998)

2. V. Garzó, J.W. Dufty, Phys. Rev. E 59(5), 58955911 (1999)

3. A. Armanini, H. Capart, L. Fraccarollo, M. Larcher, J. Fluid Mech. 532, 269-319 (2005)

4. D. Berzi, L. Fraccarollo, Geophys. Phys. Fluids 25, 106601 (2013)

5. B. Spinewine, H. Capart, J. Fluid Mech. 731, 579613 (2013)

6. V. Matoušek, V. Bareš, J. Krupička, T. Picek, Š. Zrostlík, J. Hydrol. Hydromech. 63(4), 318-326 (2015)

7. B. Spinewine, H. Capart, L. Fraccarollo, M. Larcher, Exp. Fluids 50(6), 1507-1525 (2011)

8. H. Capart, D. L. Young, Y. Zech, Exp. Fluids 32, 121-135 (2002)

9. V. Matoušek, V. Bareš, J. Krupička, T. Picek, Š. Zrostlík, Can. J. Chem. Eng. 94, 1076-1083 (2016)

10. V. Matoušek, V. Bareš, J. Krupička, T. Picek, Š. Zrostlík, River Flow 2016 (IAHR, Saint Louis, 2016)

11. V. Matoušek, Hydrotransport 2017 (BHRG, Melbourne, 2017)

12. D. Berzi, ASCE J. Hydraul. Eng. 137(10), 12001207 (2011)

13. H. Capart, L. Fraccarollo, Geophys. Res. Lett. 38. L20402 (2011) 